170

\section{DIFFUSIONE EPIDEMICA DI UN CEPPO MDR DI K. PNEUMONIAE CTX-M-I5 PRODUTTORE IN UNA TERAPIA INTENSIVA NEONATALE}

Migliavacca R.', Cavigioli F.2, Facchini M. ${ }^{3}$, Spalla M. ${ }^{4}$, Lista G. ${ }^{2}$, Nucleo E.', De Luca C.', Pagani L.'

'Dip. S.M.E.C. sez. di Microbiologia, Università di Pavia, via Brambilla 74, 27 I 00 Pavia;

¿U.O. Terapia Intensiva Neonatale, Ospedale dei Bambini

"V. Buzzi", via Castelvetro 32, 20154 Milano;

3U.O. Laboratorio Analisi, Batteriologia, Ospedale dei Bambini

"V. Buzzi", Milano, via Castelvetro 32, 20154 Milano;

${ }^{4}$ Servizio Analisi Microbiologiche Fondazione IRCCS "S. Matteo", p.le Golgi, 27l 00 Pavia;

Introduzione. Le extended-spectrum beta-lattamasi (ESßL) di tipo CTX-M sono state inizialmente descritte nella seconda metà degli anni '80 e la loro diffusione nelle Enterobacteriaceae, dal 1995 ad oggi, sta aumentando drammaticamente in molte parti del mondo. In Italia, la ES?L CTX-M-15 è stata riportata prevalentemente in E.coli e, più recentemente, in K.pneumoniae con una diffusione di tipo regionale. Ad oggi, non sono stati riportati outbreak sostenuti da K.pneumoniae produttore di CTXM-15 in reparti di Terapia Intensiva Neonatale (NICU).

Scopo del lavoro. Caratterizzare i ceppi di K.pneumoniae produttori di ESßL responsabili di un episodio epidemico avvenuto in una NICU.

Materiali e Metodi. La produzione di ESßL è stata confermata con il test CLSI (linee guida 2007).

Per la caratterizzazione e l'identificazione delle ESßL prodotte sono stati eseguiti IEF, amplificazione con primer specifici del gruppo di beta-lattamasi CTX-M 3-15-22 e sequenziamento. La tipizzazione degli isolati è stata effettuata mediante PFGE (Not1) ed i risultati valutati con il software Fingerprinting II (Bio-Rad).

Risultati. Nel periodo 24 ottobre 2006 - 2 gennaio 2007 presso la NICU dell'Ospedale Buzzi (Milano) sono stati ricoverati 118 pazienti; di questi, 20 (16.9\%) sono risultati colonizzati da un ceppo di K.pneumoniae ESßL produttore e 3 hanno sviluppato sepsi, con esisto infausto in un caso. Un ceppo con le stesse caratteristiche è stato isolato dall'olio dermatologico in uso. Gli isolati erano tutti caratterizzati da multiresistenza con sensibilità solo ai carbapenemi. Lo studio delle beta-lattamasi ha rivelato in ogni caso la produzione di due enzimi con pI 7.6 e 8.6, questo ultimo con attività idrolitica su cefotaxime. Tutti gli isolati sono risultati produttori di CTX-M-15 e tutti clonalmente correlati.

Conclusioni. I risultati di questo studio dimostrano un’ampia circolazione dei ceppi di K.pneumoniae produttori di CTX-M-15 e la necessità di una sorveglianza mirata soprattutto in reparti ad alto rischio.

\section{1}

\section{VALUTAZIONE “IN VITRO” DI TERRENI AGARIZZATI PER LA RILEVAZIONE DI CEPPI BATTERICI ESBL PRODUTTORI}

Vujinovic B., Arighi E., Botta F., Casartelli R., Moretti G., Pieretti B., Tettamanti B., Terramocci R.

Laboratorio Analisi Ospedale "Valduce", via Dante II, 22100 Como (CO)

Introduzione. In seguito alla segnalazione negli ultimi anni di un aumento della prevalenza di microrganismi produttori di ß-lattamasi ad ampio spettro (ESßL+), nasce l'esigenza di individuare tempestivamente questi ceppi batterici isolati da diversi materiali biologici, in quanto risultano associati a fallimento terapeutico (multiresistenza vs penicilline e cefalosporine) e ad un peggioramento del quadro clinico del paziente.

Scopo di questo lavoro è quello di valutare nella pratica microbiologica, in associazione ai comuni terreni di coltura utilizzati per l'isolamento dei ceppi batterici da diversi materiali biologici, la sensibilità e la specificità di terreni selettivi in grado di rilevare in prima battuta l'eventuale presenza di ceppi ESßL+.

Materiali e Metodi. Diversi materiali biologici (espettorati, broncoaspirati, broncolavaggi alveolari, urine, emocolture, ecc.) appartenenti a pazienti ospedalizzati presso l'Ospedale "Valduce" di Como e ricoverati in Case di Cura afferenti l'ospedale stesso, sono stati processati e seminati secondo le metodiche in uso nel nostro laboratorio anche sui terreni selettivi per la rilevazione presuntiva di ceppi ESßL+ [Agar chromoID ESßL (bioMérieux) e Agar ESßLA (AES Chemunex, distribuite da Biolife)].

Risultati. In un primo momento abbiamo valutato la sensibilità e la specificità dei due terreni seminando direttamente ceppi batterici di controllo ATCC e ceppi di Enterobacteriaceae ESßL+ precedentemente isolati. Questi ultimi sono stati selezionati in base alla segnalazione da parte del sistema automatico Vitek2 Compact ed alla conferma con la prova del doppio disco su terreno MuellerHinton agar secondo quanto stabilito dal CLSI. Abbiamo inoltre valutato per entrambi i terreni il fattore di diluizione minimo per la crescita dei presunti ceppi ESßL $+\left(\geq 10^{4}\right.$ $\mathrm{UFC} / \mathrm{ml}$ ). Sono stati esaminati circa 100 pazienti, per diversi materiali biologici, che all'analisi microscopica di screening risultavano positivi per la presenza di bacilli gram negativi.

Conclusioni. Dai dati raccolti è emerso che entrambi i terreni selettivi (Agar chromoID ESßL e Agar ESßLA) mostrano buona sensibilità e specificità per i ceppi batterici ESßL+, in accordo con le segnalazioni del Vitek2 Compact e la prova del doppio disco. Su entrambi i terreni crescono Pseudomonas spp. multiresistenti e ceppi di Enterobacteriaceae produttori di cefalosporinasi. Questi terreni possono essere affiancati nella routine microbiologica a quelli di uso comune per la rilevazione presuntiva di ceppi ESßL+ al fine di ridurre i tempi di attesa per una terapia antibiotica mirata, soprattutto nei pazienti critici. 